PROCEEDINGS OF THE

AMERICAN MATHEMATICAL SOCIETY

Volume 137, Number 6, June 2009, Pages 2139-2145

S 0002-9939(09)09621-X

Article electronically published on January 8, 2009

\title{
GENERIC SPECTRAL SIMPLICITY OF POLYGONS
}

\author{
LUC HILLAIRET AND CHRIS JUDGE
}

(Communicated by Matthew J. Gursky)

\begin{abstract}
We study the Laplace operator with Dirichlet or Neumann boundary conditions on polygons in the Euclidean plane. We prove that almost every simply connected polygon with at least four vertices has a simple spectrum. We also address the more general case of geodesic polygons in a constant curvature space form.
\end{abstract}

\section{INTRODUCTION}

Let $\mathcal{P}_{n}$ be the space of polygons in $\mathbb{R}^{2}$ having $n$ vertices and boundary equal to a simple closed curve. By labeling the vertices, one obtains a local parametrization of $\mathcal{P}_{n}$ by $\mathbb{R}^{2 n}$. This defines an affine structure on $\mathcal{P}_{n}$ as well as a notion of sets of measure zero.

Theorem 1.1. If $n \geq 4$, then for almost every $P \in \mathcal{P}_{n}$, the Dirichlet (resp. Neumann) Euclidean Laplacian on $P$ has one-dimensional eigenspaces.

As of yet, we do not know whether or not the generic triangle has a simple spectrum. The methods of this paper show that either the generic triangle has a simple spectrum or no triangle has a simple spectrum.

We also generalize Theorem 1.1 to

Theorem 1.2. Almost every simply connected geodesic polygon belonging to a hemisphere of the standard sphere has a simple Dirichlet (resp. Neumann) spectrum. Almost every simply connected geodesic polygon in the hyperbolic plane has a simple Dirichlet (resp. Neumann) spectrum.

It is conjectured by number theorists that the geodesic triangle in the hyperbolic plane $\mathbb{H}^{2}$ with angles $\pi / 2, \pi / 6$, and 0 has a simple spectrum Srn. The (joint) spectrum of this triangle coincides with the spectrum of the hyperbolic surface $\mathbb{H}^{2} / \mathrm{SL}(2, \mathbb{Z})$. It is not known whether there exists a closed hyperbolic surface with a simple Laplace spectrum.

We note that K. Uhlenbeck [], building upon earlier work of J. Albert A], proved the simplicity of the Laplace spectrum for the generic compact domain in $\mathbb{R}^{d}$ and the generic metric on a compact manifold. Such moduli spaces are infinite dimensional whereas $\mathcal{P}_{n}$ is only finite dimensional.

Received by the editors September 4, 2007, and, in revised form, April 22, 2008.

2000 Mathematics Subject Classification. Primary 58J50.

(C)2009 American Mathematical Society Reverts to public domain 28 years from publication 


\section{AnAlytic BI-LiPSChitz BOUNDARY PERTURBATIONS}

The proofs of Theorems 1.1 and 1.2 are based upon analytic perturbation theory. We first show that the Laplace spectrum of a real-analytic path $P_{t}$ of polygons depends real-analytically on $t$. This will follow from

Lemma 2.1. Let $\Omega_{t}, 0 \leq t \leq 1$, be a family of Lipschitz domains such that there exists a real-analytic family of homeomorphisms $f_{t}: \Omega_{0} \rightarrow \Omega_{t}$ and a constant $C>0$ such that

$$
C \cdot|x-y| \leq\left|f_{t}(x)-f_{t}(y)\right| \leq \frac{1}{C} \cdot|x-y|
$$

Then the eigenvalues of the Neumann (resp. Dirichlet) Laplacian, $\Delta_{t}$, vary realanalytically in $t$.

Proof.1 For each $t_{0}$, there exists an interval neighborhood $I \ni t_{0}$ and Lipschitz functions $f^{(k)}$ such that $f_{t}(x)=\sum_{k} f^{(k)}(x)\left(t-t_{0}\right)^{k}$. By Rademacher's theorem, for each $k$, there exists a set of full measure $A_{k} \subset \Omega_{0}$ such that for each $x \in \Omega_{0}$, the differential $D_{x} f^{(k)}$ exists. Letting $A=\bigcap_{k} A_{k}$ we obtain a set of full measure such that $D_{x} f_{t}$ exists for all $x \in A$ and $t \in I$.

From (2.1) we have $C \leq\left|D_{x} f_{t}\right| \leq C^{-1}$ for $x \in A$. It follows that the pullback operator $f_{t}^{*}: C^{\infty}\left(\Omega_{t}\right) \rightarrow C^{\infty}\left(\Omega_{0}\right)$ extends continuously in the $H^{1}$ norm to an isomorphism from $H^{1}\left(\Omega_{t}\right)$ to $H^{1}\left(\Omega_{0}\right)$. Also, it follows that the measure $\left(f_{t}\right)_{*}^{-1}(\mu)$ is absolutely continuous with respect to Lebesgue measure, and hence equals $h_{t} d \mu$ for some function $h_{t}: \Omega_{0} \rightarrow \mathbb{R}$ that is positive almost everywhere.

For any $v \in H^{1}\left(\Omega_{t}\right)$, we then have that

$$
\begin{aligned}
\int_{\Omega_{t}}|v(X)|^{2} d \mu(X) & =\int_{\Omega_{0}}\left|v \circ f_{t}(x)\right|^{2} h_{t}(x) d \mu(x), \\
\nabla_{x}\left(v \circ f_{t}\right) & =D_{x} f_{t} \cdot\left[\left(\nabla_{X} v\right) \circ f_{t}\right] .
\end{aligned}
$$

It follows that the operator $H_{t}$ defined by

$$
H_{t}(u)=\left(u \circ f_{t}\right)
$$

is unitary as a map from $L^{2}\left(\Omega_{t}, d \mu\right)$ onto $L^{2}\left(\Omega_{0}, h_{t} d \mu\right)$.

Let us consider first the Neumann eigenvalue problem: $\Delta_{t}$ is the self-adjoint operator associated with the quadratic form $q_{t}$ defined on $H^{1}\left(\Omega_{t}\right)$ by

$$
q_{t}(u)=\int_{\Omega_{0}}\left|\nabla_{x} u\right|^{2} d \mu .
$$

For each $t$, define the $\tilde{q}_{t}$ on $H^{1}\left(\Omega_{0}\right)$ by $\tilde{q}_{t}=q_{t} \circ H_{t}^{-1}$. Using (2.2) and (2.3) we find that

$$
\tilde{q}_{t}(u)=\int_{\Omega_{0}}\left|\left(D_{x} f_{t}\right)^{-1} \nabla_{x} u\right|^{2} h_{t} d \mu .
$$

Denote by $\tilde{\Delta}_{t}$ the associated self-adjoint operator (with respect to $L^{2}\left(\Omega_{0}, h_{t} d \mu\right)$ ). Since $H_{t}$ defines a unitary equivalence, the operators $\tilde{\Delta}_{t}$ and $\Delta_{t}$ have the same Neumann spectrum.

Using the power series expansion for $f_{t}$ in $t$, we find that, for each $u \in H^{1}\left(\Omega_{0}\right)$, the maps $t \mapsto \tilde{q}_{t}(u)$ and $t \mapsto \int_{\Omega_{0}}|u|^{2} h_{t} d \mu$ are real-analytic. The spectrum of $\tilde{\Delta}_{t}$ is

\footnotetext{
${ }^{1}$ In the case where $f_{t}$ is a real-analytic family of smooth diffeomorphisms, this result is standard in the theory of boundary perturbations. See, for instance, $\mathrm{K}$ §VII.6.5]. Our proof will follow the same lines.
} 
thus determined by a generalized eigenvalue problem in the sense of Kato (see $\underline{K}$. $\S$ VII.6]). The conclusion thus holds for the Neumann problem.

In the case of Dirichlet eigenvalues, $\Delta_{t}$ is the self-adjoint operator associated with $q_{t}$ defined on $H_{0}^{1}\left(\Omega_{t}\right)$, the closure in $H^{1}\left(\Omega_{t}\right)$ of the smooth functions on $\Omega_{t}$ that vanish on the boundary of $\Omega_{t}$. Since $H_{t}$ maps $H_{0}^{1}\left(\Omega_{t}\right)$ onto $H_{0}^{1}\left(\Omega_{0}\right)$ unitarily, the same method of proof applies.

Corollary 2.2. If for some $t_{0}$, the spectrum of $\Delta_{t_{0}}$ is simple, then for all but countably many $t$, the spectrum of $\Delta_{t}$ is simple.

Proof. Let $\lambda_{n}(t)$ be the real-analytic functions corresponding to the eigenvalues of $\Delta_{t}$. Since the spectrum of $\Delta_{t_{0}}$ is simple, $\lambda_{i}\left(t_{0}\right) \neq \lambda_{j}\left(t_{0}\right)$ if $i \neq j$. Thus, since they are real-analytic, the set $\left\{t \mid \lambda_{i}(t)=\lambda_{j}(t)\right\}$ is at most countable for every $i \neq j$. A countable union of countable sets is countable.

In what follows, we will consider linear families of piecewise linear homeomorphisms. These are real-analytic families of uniformly bi-Lipschitz homeomorphisms that describe all deformations of polygons. Corollary 2.2 thus implies the following proposition.

Proposition 2.3. If the Laplace spectrum of $P \in \mathcal{P}^{n}$ is simple, then there exists an open ball neighborhood $\mathcal{B}$ of $P \in \mathcal{P}_{n} \subset \mathbb{R}^{2 n}$ such that almost every polygon in $Q \in \mathcal{B}$ has a simple spectrum.

Proof. Triangulate $P$ so that the vertices of the triangulation that lie in $\partial P$ are exactly the vertices of $P$. Let $v_{n+1}, \ldots, v_{n+k}$ denote the additional vertices. Let $\mathcal{B} \subset \mathbb{R}^{2 n}$ be a ball neighborhood of $P=\left(v_{1}, v_{2}, \ldots, v_{n}\right)$ such that for each $P^{\prime}=$ $\left(v_{1}^{\prime}, v_{2}^{\prime}, \ldots, v_{n}^{\prime}\right) \in \mathcal{B}$, we have that $P^{\prime}$ is a simply connected polygon and $v_{1}^{\prime}, v_{2}^{\prime}, \ldots$, $v_{n}^{\prime}, v_{n+1}, \ldots, v_{n+k}$ are the vertices of a triangulation of $P^{\prime}$. Using the triangulation, we can define a piecewise linear homeomorphism $f$ such that $P^{\prime}=f(P)$. By letting $f_{t}=(1-t) \mathrm{Id}+t f$ and $P_{t}=f_{t}(P)$, we obtain a linear family of piecewise linear homeomorphisms corresponding to the segment in $\mathcal{P}^{n}$ that joins $P$ to $P^{\prime}$.

Thus, by Corollary [2.2, for all but countably many $t$, the spectrum of $\Delta_{P_{t}}$ is simple. In other words, for each radial line segment $\sigma$ based on $P$ and lying in $\mathcal{B}$, the set of $Q \in \sigma$ that do not have a simple spectrum is countable and hence this set has measure zero. The claim then follows from the use of polar coordinates based at $P$ and Fubini's theorem.

\section{Proof of Theorem 1.1}

Proposition 2.3 reduces the proof of Theorem 1.1 to verifying that for each $n \geq 4$, the set $\mathcal{P}^{n} \subset \mathbb{R}^{2 n}$ is connected and contains at least one polygon $P$ with a simple spectrum. We first show that for each $n \geq 4$ there exists an $n$-gon whose Laplace spectrum is simple.

Let $R$ be a rectangle with sidelengths $s_{1}$ and $s_{2}$. An $L^{2}(R)$ basis of Neumann (resp. Dirichlet) Laplace eigenfunctions of $R$ is given by $\cos \left(\pi m x / s_{1}\right) \cdot \cos \left(\pi n x / s_{2}\right)$ (resp. $\left.\sin \left(\pi m x / s_{1}\right) \cdot \sin \left(\pi n x / s_{2}\right)\right)$, where $m$ and $n$ vary over the nonnegative integers (resp. positive integers). In particular, we have multiple eigenvalues iff there exist pairs of integers $(m, n)$ and $(\bar{m}, \bar{n})$ so that

$$
\frac{m^{2}}{s_{1}^{2}}+\frac{n^{2}}{s_{2}^{2}}=\frac{\bar{m}^{2}}{s_{1}^{2}}+\frac{\bar{n}^{2}}{s_{2}^{2}}
$$


Thus if $\left(s_{1} / s_{2}\right)^{2} \notin \mathbb{Q}$, then the spectrum of the rectangle $R$ is simple.

By adding $n-4$ vertices to a side of a rectangle, one may regard any rectangle as an $n$-gon. The spectrum is unchanged by adding such 'false' vertices. Thus, for any $n \geq 4$, there exists an $n$-gon with a simple spectrum.

The (path) connectedness of $\mathcal{P}^{n}$ can be verified by induction on the number of vertices 2 The case of three vertices can be verified in many ways. For example, the connected Lie group consisting of affine homeomorphisms of $\mathbb{R}^{2}$ acts transitively and continuously on triples of points in $\mathbb{R}^{2}$ that are the vertices of a nondegenerate triangle.

Recall that an $(n+1)$-gon may be regarded as an $n$-gon if three successive vertices lie on the same line segment. We will show that any $(n+1)$-gon, $n \geq 3$, can be linearly deformed into an $n$-gon. Thus, the claim will follow from the induction hypothesis.

Any $(n+1)$-gon $P=\left(v_{1}, \ldots, v_{n+1}\right)$ can be triangulated so that the vertices of the triangulation are exactly the vertices $v_{1}, \ldots, v_{n+1}$ of the polygon 3 Since $P$ is simply connected and $n+1 \geq 4$, the dual graph of this triangulation is a tree with at least two vertices. Let $T$ be a triangle corresponding to an end of the dual graph. Then there is a vertex $v$ of $T$ such that both of the sides of $T$ adjacent to $v$ are also sides of $P$. Without loss of generality, $v=v_{1}$.

Let $m$ be the midpoint (for example) of the side of $T$ that is opposite to $v_{i}$. Define

$$
P_{t}=\left((1-t) \cdot v_{1}+t \cdot m, v_{2}, \ldots, v_{n+1}\right) .
$$

Note that the vertex $m$ lies in a segment joining two other vertices of $P_{1}=$ $\left(m, v_{2}, \ldots, v_{n+1}\right)$. Hence $P_{1}$ can be regarded as an $n$-gon.

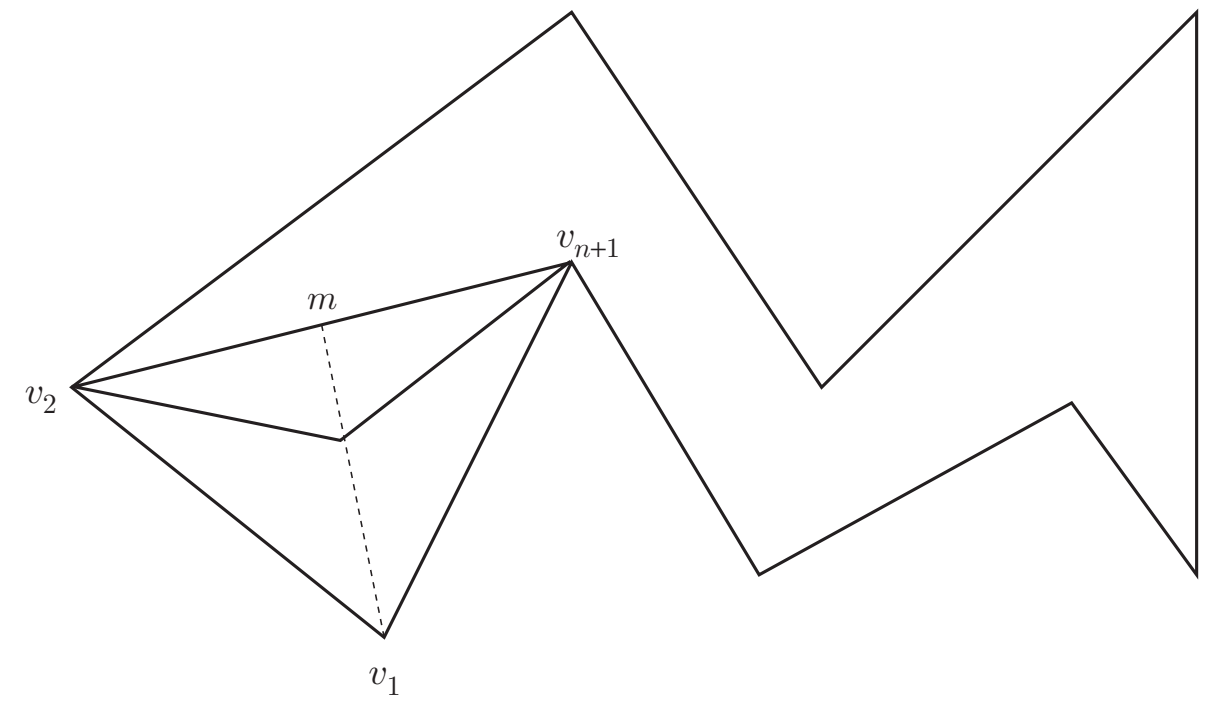

Figure 1. Deleting a vertex

\footnotetext{
${ }^{2}$ We remark that any star-shaped $n$-gon can be easily connected to a rectangle with $n-4$ 'false' vertices.

${ }^{3}$ For example, the Delaunay triangulation of $P$ has this property $\mathrm{Thr}$.
} 


\section{Proof of Theorem 1.2}

In this section we prove Theorem 1.2. The key is to construct, for each Euclidean polygon $P_{0}$, a natural real-analytic family of polygons $\kappa \rightarrow P_{\kappa}$ such that $P_{\kappa}$ is a geodesic polygon in the constant curvature $\kappa$ space form $M_{\kappa}$. To make the construction transparent, we make a convenient choice of model for $M_{\kappa}$.

The following constructions are standard. (See for example [T].) For $R>0$, let

$$
\tilde{M}_{R}^{ \pm}=\left\{(X, Y, Z) \mid X^{2}+Y^{2} \pm Z^{2}= \pm R^{2}\right\},
$$

and let $M_{R}^{ \pm}$be the connected component of $\tilde{M}_{R}^{ \pm}$that contains the point $(0,0, R)$. The quadratic form $X^{2}+Y^{2} \pm Z^{2}$ defines a Riemannian metric of constant curvature $\pm R^{-2}$ on $M_{R}^{ \pm}$. For example, $M_{1}^{-}$is isometric to the hyperbolic plane and $M_{1}^{+}$is the unit sphere.

We construct a projective (Klein) model as follows: Given a point $P \in M_{R}^{ \pm}$, there exists a unique pair $(X, Y)$ such that $(X, Y, R)$ lies in the line containing $P$ and the origin. This defines an injective map $F_{R}^{-}: M_{R}^{-} \rightarrow \mathbb{R}^{2}$ and a two-to-one map $F_{R}^{+}: M_{R}^{+} \rightarrow \mathbb{R}^{2}$. Henceforth, we will restrict $F_{R}^{+}$to the upper hemisphere of $M_{R}^{+}$. By pulling the constant curvature metrics back by the diffeomorphism $\left(F_{R}^{ \pm}\right)^{-1}$ and by setting $\kappa= \pm R^{-2}$, we obtain the following model for $M_{\kappa}$ :

Proposition 4.1. Let $(\rho, \theta)$ be polar coordinates on $\mathbb{R}^{2}$ and let

$$
g_{\kappa}=\frac{1}{\left(1+\kappa \cdot \rho^{2}\right)^{2}} d \rho^{2}+\frac{\rho^{2}}{1+\kappa \cdot \rho^{2}} d \theta^{2} .
$$

If $\kappa<0$, then $g_{\kappa}$ is a complete Riemannian metric of constant curvature $\kappa$ on the Euclidean disc of radius $R=|\kappa|^{-\frac{1}{2}}$. If $\kappa \geq 0$, then $g_{\kappa}$ is a complete Riemannian metric of constant curvature $\kappa$ on the Euclidean plane.

Remark 4.2. Note that a path is geodesic with respect to $g_{\kappa}$ if and only if it is a Euclidean line segment. (Indeed, each geodesic in $M_{R}^{ \pm}$is the intersection of a plane through the origin and $F_{R}^{ \pm}$is defined via radial projection.) In particular, given $\kappa, \kappa^{\prime} \in \mathbb{R}$, a curve in $\mathbb{R}^{2}$ is a geodesic polygon with respect to $g_{\kappa}$ if and only if it is a polygon with respect to $g_{\kappa^{\prime}}$ and the obvious containment conditions are satisfied.

Let $P$ be a Euclidean polygon belonging to the Euclidean ball $B(0, R)$. Let $\nabla_{\kappa}$ (resp. $d v_{\kappa}$ ) denote the gradient (resp. volume form) associated to $g_{\kappa}$. Define the quadratic form

$$
q_{\kappa}(u)=\int_{P}\left|\nabla_{\kappa} u\right|^{2} d v_{\kappa} .
$$

Let $\Delta_{\kappa}$ denote the self-adjoint operator associated with $q_{\kappa}$ defined either on $H_{0}^{1}(P)$ (Dirichlet boundary condition) or on $H^{1}(P)$ (Neumann boundary condition).

Proposition 4.3. The eigenvalues of $\Delta_{\kappa}$ vary analytically for $\kappa \in\left(-R^{-2}, \infty\right)$.

Proof. For each compact interval in $\left(-R^{-2}, \infty\right)$, uniform estimates on the coefficients of $g_{\kappa}$ on $P$ provide $C>0$ such that $C^{-1}\left|\nabla_{0} f\right|_{0} \leq\left|\nabla_{\kappa} f\right|_{\kappa} \leq C\left|\nabla_{0} f\right|_{0}$ and $C^{-1} \leq d v_{\kappa} / d v_{0} \leq C$. It follows that the form domain of $q_{\kappa}$ is independent of $\kappa$. Inspection of (4.1) shows that the metric $g_{\kappa}$ depends analytically on $\kappa \in\left(-R^{-2}, \infty\right)$. It follows that both $q_{\kappa}(u)$ and the quadratic form $u \rightarrow \int|u|^{2} d v_{\kappa}$ depend analytically on $\kappa$. Kato-Rellich theory thus applies, yielding the proposition.

Theorem 1.2 will follow from 
Theorem 4.4. For any $\kappa$, almost every simply connected, geodesic polygon in $M_{\kappa}$ has a simple Neumann (resp. Dirichlet) spectrum.

Proof. By Remark 4.2 we can identify the set of geodesic $n$-gons in $M_{\kappa}$ with a set of Euclidean polygons. In particular, the set of all geodesic polygons in $M_{\kappa}$ with $\kappa \leq 0$ can be identified with the set $U \subset \mathcal{P}_{n} \times(-\infty, 0]$ defined by

$$
U=\left\{(P, \kappa) \mid \kappa \leq 0, P \subset B\left(0,|\kappa|^{-\frac{1}{2}}\right)\right\} .
$$

By Theorem 1.1 and Proposition 4.3. for almost every $P \in \mathcal{P}_{n}$, the set of $\kappa$ such that $(P, \kappa) \in U$ has a simple spectrum is at most countable. (See the proof of Corollary 2.2.) It follows that, with respect to Lebesgue measure on $\mathcal{P}_{n} \times \mathbb{R}$, the spectrum of almost every $(P, \kappa) \in U$ is simple.

Given $\kappa \leq 0$, let $A_{\kappa}$ be the set of $P \in \mathcal{P}_{n}$ such that $(P, \kappa) \in U$ does not have a simple spectrum. Then it follows from above that for almost every $\kappa \in \mathbb{R}$, the set $A_{\kappa}$ has measure zero.

To conclude that the measure of $A_{\kappa}$ equals zero for every $\kappa$, we use the fact that if a metric $g$ is rescaled by a constant $k$, then the Laplacian is rescaled by $k^{-1}$. In particular, the multiplicity of each eigenvalue does not change if the metric is rescaled.

On the other hand, the curvature of the metric does change under rescaling if the initial curvature is nonzero. In particular, the metric $\left(\kappa / \kappa^{\prime}\right) \cdot g_{\kappa}$ on $B\left(0,|\kappa|^{-\frac{1}{2}}\right)$ has curvature $\kappa^{\prime}$ and serves as another model for $M_{\kappa^{\prime}}$. It follows that if $A_{\kappa}$ has measure zero, then so does $A_{\kappa^{\prime}}$.

The case of nonnegative curvature is proven similarly.

\section{ACKNOWLEDGMENTS}

We thank the Fields Institute for hosting a workshop where this work began. We also thank Gilles Carron and David Hoff for helpful discussions. Finally, we thank the referee for a careful reading of the manuscript.

\section{REFERENCES}

A. Albert, J. H., Genericity of simple eigenvalues for elliptic PDE's. Proc. Amer. Math. Soc. 48 (1975), 413-418. MR0385934 (52:6793)

CH. Courant, R. and Hilbert, D., Methods of mathematical physics. I, John Wiley \& Sons, Inc., New York, 1989. MR0065391 (16:426a)

K. Kato, T., Perturbation theory for linear operators. Springer-Verlag, Berlin, 1995. MR.1335452 (96a:47025)

Srn. Sarnak, P., Spectra of hyperbolic surfaces. Bull. Amer. Math. Soc. (N.S.) 40 (2003), no. 4, 441-478. MR 1997348 (2004f:11107)

S. Semmes, S., Analysis on singular spaces, an appendix in Metric structures for Riemannian and non-Riemannian spaces by M. Gromov, Progress in Mathematics, vol. 152. Birkhäuser, Boston, 1999. MR1699320(2000d:53065)

Thr. Thurston, W. P., Shapes of polyhedra and triangulations of the sphere. The Epstein birthday schrift, 511-549, Geom. Topol. Monogr., 1, Geom. Topol. Publ., Coventry, 1998. MR.1668340 (2000b:57026) 
T. Thurston, W. P., Three-dimensional geometry and topology, Princeton Univ. Press, 1997. MR $1435975(97 \mathrm{~m}: 57016)$

U. Uhlenbeck, K., Generic properties of eigenfunctions. Amer. J. Math. 98 (1976), no. 4, 10591078. MR0464332(57:4264)

Laboratoire de Mathématiques Jean Leray, UMR CNRS 6629-Université de Nantes, 2 Rue de la Houssinière, BP 92 208, F-44 322 Nantes Cedex 3, France

E-mail address: Luc.Hillairet@math.univ-nantes.fr

Department of Mathematics, Indiana University, Bloomington, Indiana 47401

E-mail address: cjudge@indiana.edu 